\title{
RETRACTED_Four-weeks intervention with jaboticaba peel lowers MDA levels after post-prandial challenge in healthy adults
}

\author{
Â.G. Batista ${ }^{1,2 *}$,Y.C. Zanzer ${ }^{1 *}$, M.R. Maróstica Júnior ${ }^{2}$ and E.M. Östman ${ }^{1}$ \\ ${ }^{1}$ Food for Health Science Centre, Lund University, P.O. Box 124, SE-22100 Lund, Sweden and \\ ${ }^{2}$ School of Food Engineering, University of Campinas, Campinas-SP, Brazil
}

Oxidative stress could play a causative role towards insulin resistance, inflammation and obesity-associated metabolic syndrome ${ }^{(1)}$. Consumption of berries such as Myrciaria jaboticaba, which is rich in anthocyanins and ellagitanins has been associated with health benefits, for instance lowering hepatic and brain oxidative stress by decreasing lipid peroxidation ${ }^{(2,3)}$. In the present study we aimed to investigate whether 4-weeks intervention of jaboticaba peel (JP) could modulate the postprandial lipid peroxidation, as well as basal metabolic rate (BMR), fat mass (FM) and free-fat mass (FFM). This work was approved by Lund University Ethics Committee (Dnr. 2015/390)

Eighteen healthy adults ( 7 men; 11 women; age $27.5 \pm 1.13$ years; body mass index $24.69 \pm 0.58 \mathrm{~kg} . \mathrm{m}^{-2}$ ) completed $4-\mathrm{weeks}$ randomized, controlled, cross-over intervention with 28 days washout period between treatments. Participants were randomized to consume $150 \mathrm{ml}$ of water blended with either JP (250 mg polyphenols, $1.8 \mathrm{~g}$ sugar and $2.4 \mathrm{~g}$ dietary fibers) or control ( $1.8 \mathrm{~g}$ sugar and $2.4 \mathrm{~g}$ dietary fibers). During the intervention and wash-out periods participants were instructed to maintain their normal diet and avoid anthocyanins, ellagic-acid, flavonoids and phenols-rich foods (according to a list provided by the study leader). Before and after 4-weeks, participants should come to our clinical facility to have post-prandial study. Venous blood samples were collected at fasting $(-30)$ and at 30,60, 120 and $180 \mathrm{~min}$ after breakfast challenge (50 g available carbohydrates) together with either JP or control drinks. MDA was assessed as a marker of lipid peroxidation in serum using a colorimetric method ${ }^{(2)}$. In addition, BMR, FM and FFM were assessed using body composition analyzer. Mixed-model analysis of covariance (PROC MIXED) with repeated measures was employed.

The MDA fasting values did not differ after 4-weeks intervention either with the control or JP $(P=0.993)$. Changes in serum MDA-levels were affected by the treatment $(P=0.003)$ but had no time $(P=0.293)$ and time $\times$ treatment interaction $(P=0.437)$. Based on least square means, the JP intervention significantly lowered serum MDA levels by $8 \cdot 3 \%$ compared to the control. No changes were observed in BMR $(P=0 \cdot 153)$, FM $(P=0 \cdot 200)$ or FFM $(P=0 \cdot 144)$.

(a)

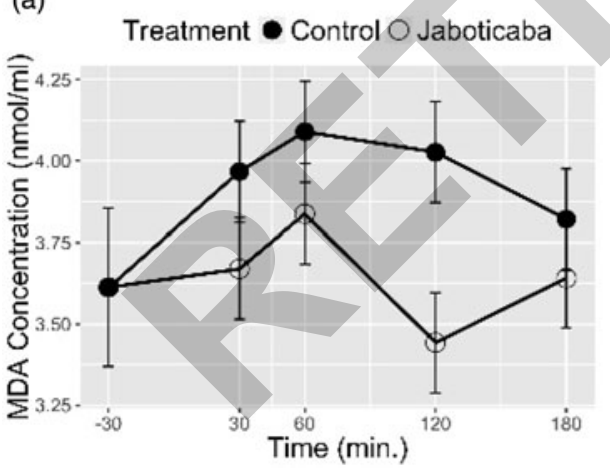

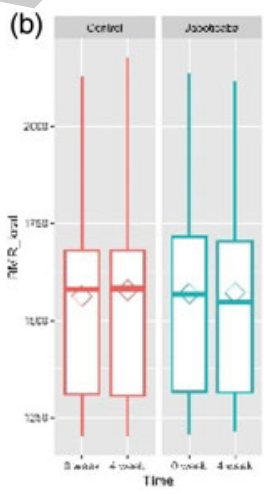

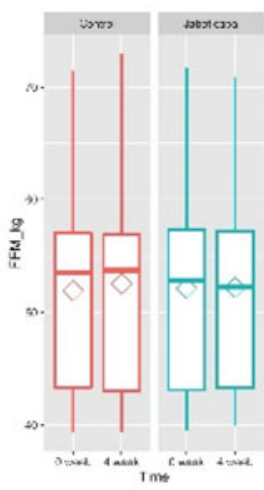

Fig. 1. (a) Postprandial MDA concentration after breakfast challenge on the last-day of 4-weeks intervention. (b) BMR, FM and FFM before and after 4 -weeks intervention.

To our knowledge, this is the first study addressing effects of JP intervention on oxidative stress and body composition in humans. We conclude that 4-weeks intake of JP reduced postprandial oxidative stress as indicated by lower MDA levels without affecting BMR, FM and FFM.

This work was supported by Brazilian foundation CNPq (Grant no. 206092/2014-8), Swedish Research Council-Swedish Research Links Prog. (Grant no. 348-2012-6189) and Food for Health Science Centre, Lund University.

1. Furukawa S, Fujita T, Shimabukuro $\mathrm{M}$ et al. (2004) J Clin Invest 114(12), 1752-1761.

2. Batista ÂG, Lenquiste SA, Cazarin CBB et al. (2014) J Funct Foods 6, 450-461.

3. Leite-Legatti AV, Batista ÂG, Dragano NRV et al. (2012) Food Res Int 49, 596-603.

*These authors contributed equally. 